\title{
Ecchymosis in critical coronavirus disease 2019 (COVID-19) patient in Tangerang, Indonesia: a case report
}

\author{
Allen Widysanto $^{1}$ (D) . T. D. Wahyuni ${ }^{1} \cdot$ L. H. Simanjuntak ${ }^{1} \cdot$ S. Sunarso ${ }^{1} \cdot$ S. S. Siahaan ${ }^{1} \cdot$ H. Haryanto $^{1} \cdot$ C. O. Pandrya $^{1}$. \\ R. C. A. Aritonang ${ }^{1}$. C. Gunawan ${ }^{2}$ (D) Angela $^{1}$ (D)
}

Accepted: 9 November 2020 / Published online: 17 November 2020

(c) Springer Science+Business Media, LLC, part of Springer Nature 2020

\begin{abstract}
We have read with great interest the articles regarding cutaneous manifestations in Coronavirus Disease 2019 (COVID-19) infection. Studies showed $20.4 \%$ of COVID-19 patients developed cutaneous manifestations. COVID-19 causes vascular endothelial injury, vasculitis and prothrombotic state which might be the underlying cause of hemorrhagic cutaneous manifestations, disseminated intravascular coagulation (DIC), and multiple organ failures. To date, hemorrhagic cutaneous manifestations reported are chilblain-like lesion, purpura to ecchymosis, livedo reticularis, and dry gangrene. We present a case of ecchymosis in COVID-19-positive ICU hospitalized patient.
\end{abstract}

Keywords COVID-19 · Cutaneous manifestation · DIC · Ecchymosis · Vasculopathy

\section{Highlights}

- Coronavirus Disease 2019 (COVID-19) is associated with vasculitis and prothrombotic state.

- Non-critical and critically ill COVID-19 patients might developed bleeding cutaneous manifestations such as purpura or ecchymosis.

- The pathophysiology of ecchymosis in COVID-19 might be caused by microvascular injury and thrombosis, vasculitis, and disseminated intravascular coagulation (DIC).

- Secondary antiphospholipid syndrome (APS) might presented in critical COVID-19 patients.

- The presence of ecchymosis in COVID-19 patients is indicating an occuring coagulopathy and associated with increased mortality in COVID-19 infection.

Allen Widysanto

allenwidy@yahoo.co.id

Siloam Hospitals Kelapa Dua, Tangerang, Indonesia

2 Faculty of Medicine, Pelita Harapan University, Tangerang, Indonesia

\section{Introduction}

Coronavirus Disease 2019 (COVID-19) is a public health emergency of international concern, including in Indonesia. Studies showed COVID-19 causes vasculitis and prothrombotic state which might be the underlying cause of some cutaneous manifestations such as purpuric rash and livedo reticularis, DIC, and multiple organ failures [1-3].We would like to report a case of ecchymosis in COVID-19-positive ICU hospitalized patient from Indonesia.

\section{Case}

A 69-years-old male patient was admitted to our hospital on March 23, 2020 with presenting symptoms after trip abroad including fever of 11 days, cough, dyspnea, and headache. The patient has history of hypertension on therapy. The vital signs and physical examinations were as follows: blood pressure $130 / 70 \mathrm{mmHg}$, pulse rate $91 /$ minute, respiratory rate $23 / \mathrm{min}$, temperature $37.2{ }^{\circ} \mathrm{C}, \mathrm{SpO} 296 \%$ on $\mathrm{O} 23$ liter per min (lpm) via nasal cannula(NC), and ronchi were found on bibasal lung fields. Chest CT scan showed mixed typed diffuse ground glass opacity and multifocal crazy paving pattern involving both lungs, predominantly in peripheral, thick-walled septated cavity on right lung subpleura, and minimal pleural effusion in segment 10 of right lung. 

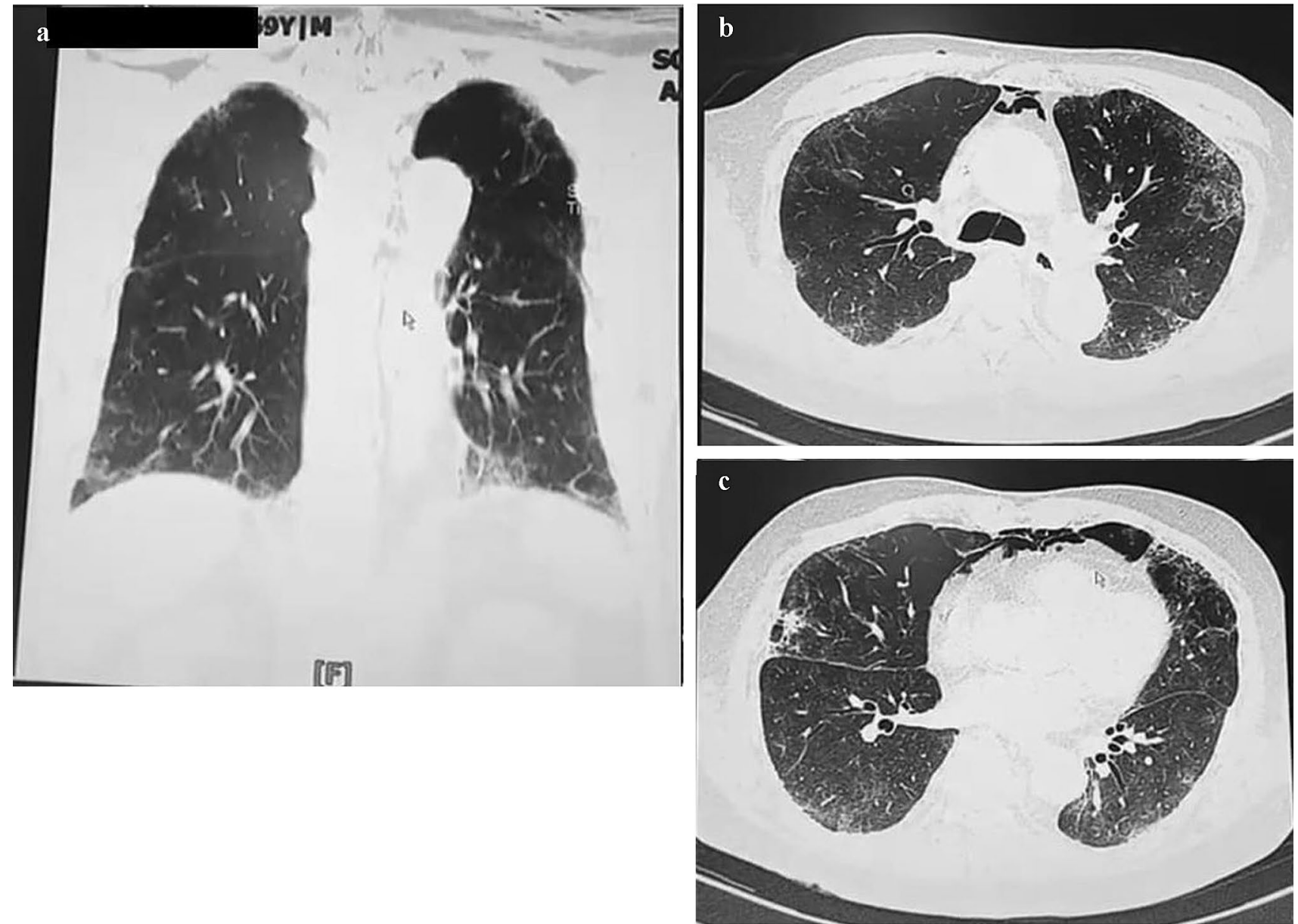

Fig. 1 Serial chest Xray a on admission showed bilateral lung opacities on all lung fields, predominantly in peripheral, and cardiomegaly with cardiothoracic ratio (CTR) $62 \%$, b worsening lung opacities, c

Chest Xray showed bilateral lung opacities on all lung fields, predominantly in peripheral, and cardiomegaly with cardiothoracic ratio (CTR) 62\% (Fig. 1). The result of the examinations on admission as follows: increased neutrophil, monocyte, erythrocyte sendimentation rate (ESR), AST, lactate dehydrogenase (LDH), C-reactive protein (CRP), and lymphocytopenia. Arterial blood gas (ABG) analysis on admission showed $\mathrm{pH} 7.436, \mathrm{PO} 248.2 \mathrm{mmHg}, \mathrm{PCO} 2$ $30.2 \mathrm{mmHg}$, HCO3 $19.9 \mathrm{mmol} / \mathrm{L}$, total CO2 $20.8 \mathrm{mmol} / \mathrm{L}$, base excess (BE) $-3 \mathrm{mmol} / \mathrm{L}, \mathrm{SpO} 286 \%$. His RT-PCR for COVID was positive, therefore he was diagnosed with COVID-19 with respiratory failure type 1 and ARDS. The patient was immediately hospitalized in ICU, given simple mask with $\mathrm{O} 26 \mathrm{lpm}$, and central venous catheter (CVC) was inserted. Next ABG showed PO2 $55.1 \mathrm{mmHg}$, therefore the simple mask was replaced by non-rebreathing mask (NRM) with $\mathrm{O} 215 \mathrm{lpm}$. The patient was treated with azithromycin, hydroxychloroquine, cefoperazone-sulbactam, tocilizumab, high dose vitamin $\mathrm{C}$, montelukast sodium, kabimidin, improvement of lung opacities, $\mathbf{d}$ the latest chest xray before hospital discharge showed improvement of lung opacities

mecobalamine, acetylcysteine, fluconazole, omeprazole, antihypertension drug, roncuronium, midazolam, morphine, methylpredisonolon, salbutamol and bromhexine $\mathrm{HCl}$.

The result of the examinations on day 4 of hospitalization (March 26) showed increased total leukocyte, neutrophil, ESR, very high D-dimer ( $>35.2 \mu \mathrm{g} / \mathrm{mL})$, AST, ALT, direct bilirubin, lymphocytopenia and low albumin. Therefore, intravenous heparin 250 units/hour was started on March 28 and continued for 7 days until April 8. However on day 7 of hospitalization (March 29), serial ABG showed pH 7.349, PO2 $121.8 \mathrm{mmHg}, \mathrm{PCO} 243.6 \mathrm{mmHg}, \mathrm{HCO} 3$ $23.5 \mathrm{mmol} / \mathrm{L}$, total $\mathrm{CO} 224.8 \mathrm{mmol} / \mathrm{L}, \mathrm{BE}-2.2 \mathrm{mmol} / \mathrm{L}$, $\mathrm{SpO} 298.5 \%$, despite treated with NRM $15 \mathrm{lpm}$, therefore, the patient was intubated and on mechanical ventilation with positive end-expiratory pressure (PEEP) 10 $\mathrm{cmH}_{2} \mathrm{O}$ and $\mathrm{FiO} 265 \%$. Patient was clinically improved everyday and on day 17 of hospitalization (April 8), the patient fulfilled the mechanical ventilation weaning criteria, therefore extubation was done and replaced by simple 

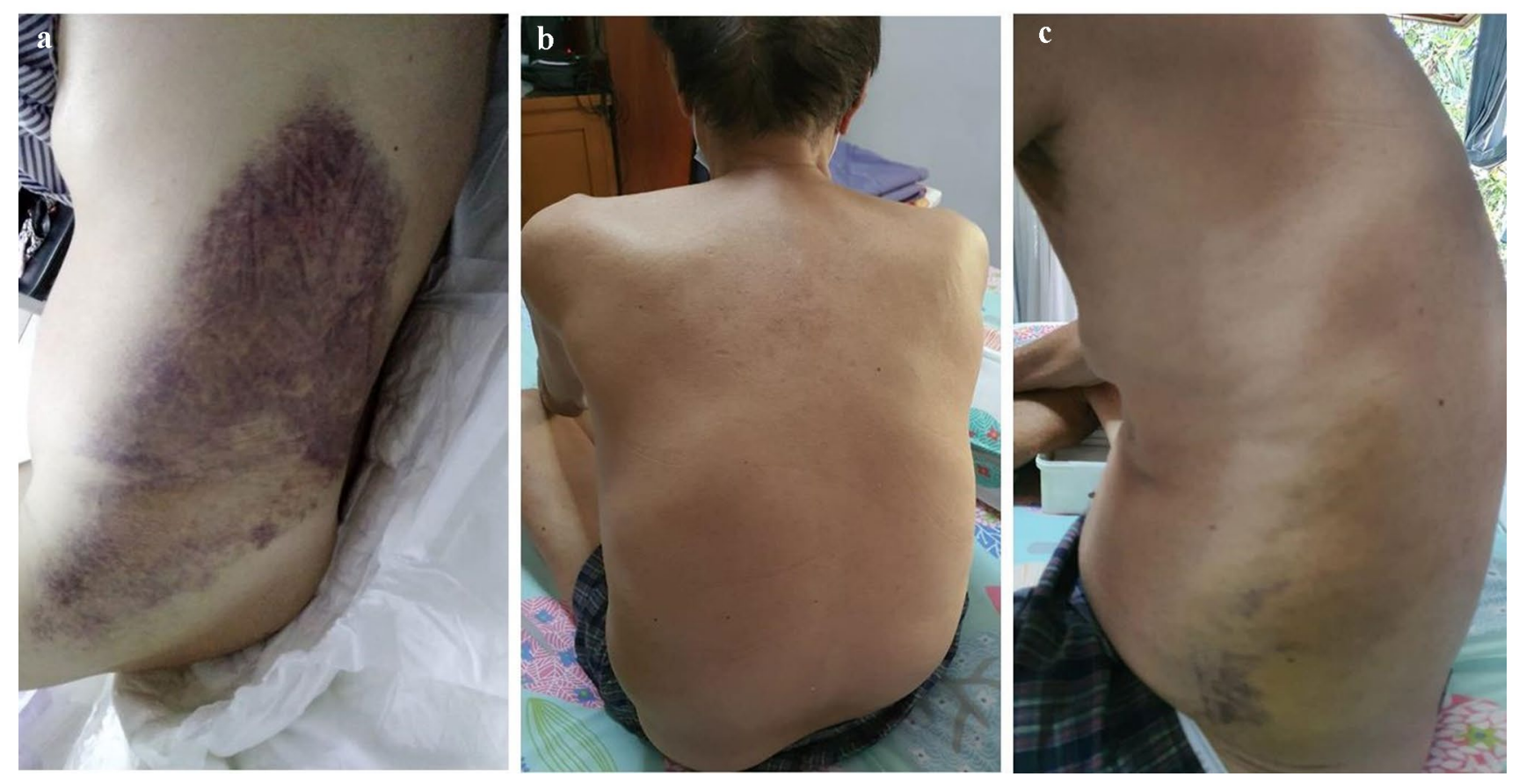

Fig. 2 a Ecchymosis involving patient's back and flank on day 25 of hospitalization (April 16), b, c Residual ecchymosis involving patient's flank during follow up on the next week after hospital discharge

mask with $\mathrm{O} 210 \mathrm{lpm}$. On the next day, simple mask was replaced by $\mathrm{NC}$ with $\mathrm{O} 24$ and 3 days later, patient was moved to HCU. Laboratory examinations on April 10 were as follows: prolonged PT and APTT, increased D-dimer, INR, total leukocyte, neutrophil, monocyte, direct bilirubin, neutrophil/lymphocyte ratio, lymphocytopenia, low haemoglobin and albumin, which showed DIC based on ISTH Score of 5 (without fibrinogen measurement).

On day 25 of hospitalization (April 16), the patient suddenly developed non-palpable and painless ecchymosis involving the back and flank (Fig. 2). However, no ecchymosis triggers other than endothelial cell injury, vasculitis, and DIC caused by COVID-19 infection were found, as there were no history trauma on the back nor pressure ulcer, because the patient was in good condition, no prolonged bed rest, and could mobilize independently during the care in HCU. The bleeding parameters were normal (PT $8.1 \mathrm{~s}$, APTT $31.7 \mathrm{~s}$, INR 0.81), except increased d-dimer $(2.91 \mu \mathrm{g} / \mathrm{mL})$. The suspicion of ecchymosis caused by the heparin could be eliminated by the fact his ecchymosis appeared 8 days after the discontinuation of heparin treatment, meanwhile, bleeding as a side effect of heparin occured during the heparin treatment until $24 \mathrm{~h}$ of heparin discontinuation. Ecchymosis caused by heparin-induced thrombocytopenia could be eliminated too because the patient had normal platelet count. Patient continued to improve clinically and so was the ecchymosis, therefore he was discharged from hospital on day
31 of hospitalization (April 22, 2020). The latest laboratory examinations showed improved, but still increased D-dimer with normal PT, APTT, and INR. During follow up on the next week, the patient has no residual symptoms besides residual ecchymosis (Fig. 2a, b).

\section{Conclusion}

This report was consistent with previous studies, which showed non-critical and critically ill COVID-19 patients might developed bleeding cutaneous manifestations such as purpura or ecchymosis. Biopsy was not done on the skin lesion in this case because of the equipment limitation, therefore, further study with biopsy is recommended. The pathophysiology of ecchymosis in this case might be described by 3 hypotheses. Firstly, previous study found the biopsy of purpuric skin rash in crititical COVID-19 patients showed pathology involving microvascular injury and thrombosis. There were also perivascular neutrophilia, lymphocytic infiltrate, and leukocytoclasia such as in pathology of vasculitis. This thrombogenic vasculopathy is caused by the deposition of C5b-9, C4d, and co-localization of COVID-19 spike (S) glycoproteins in the microvascular endothelial cell throughout the dermis in both lesion and normal-appearing skin. These cause activation of the alternative pathway (AP) and lectin pathway (LP) of complement system, resulting in microvascular endothelial cell injury, 
inflammation, and subsequent activation of the clotting pathway, leading to fibrin deposition and thrombosis [3]. Besides causing endothelial cell injury, vasculitis, and prothrombotic state, vascular response to hypoxia which is controlled by the hypoxia-inducible transcription factors (HIFs) in severe COVID-19 infection such as in this case, might aggravates thrombus formation [4]. These resulted in increased fibrin degradation product (FDP) and D-dimer, prolonged prothrombin time (PT) and activated partial thromboplastin time (APTT), which might the underlying cause of DIC as a common complication of COVID-19. Studies revealed that $71.4 \%$ of non-survivors of COVID-19 matched the grade of overt disseminated intravascular coagulation (DIC) $(\geq 5$ points according to the International Society on Thrombosis and Haemostasis criteria / ISTH).

Secondly, the ecchymosis might be associated with bradykinin in Kinin-kallikrein system (KKS) in conjunction with ACE2. The KKS consists of high-molecular-mass kininogen (HMMK) that produce bradykinin (BK) and [desArg973]-BK (DABK-the active metabolite of bradykinin). SARS-CoV-2 enters human's cells by binds to ACE2 proteins and downregulates them, meanwhile, ACE2 is important for hydrolyzing the active bradykinin metabolite DABK. Decreased activity of ACE2 impairs the inactivation of DABK and therefore, lead to reactive oxygen species formation and interference with antioxidant and vasodilatory signals such as NOX2 and eNOS, and complement activation, therefore resulting in inflammation, microvascular endothelial cell injury, vasodilation, increased capillary permeability, increase risk of ARDS, lung injury, and multiple organ failure, and thrombosis $[3,5]$.

Thirdly, ecchymosis might be caused by dermal thrombotic vasculopathy due to secondary antiphospholipid syndrome (APS). Antiphospholipid antibodies such as anticardiolipin $\operatorname{IgA}$ antibodies, anti- $\beta 2$-glycoprotein I IgA and $\operatorname{IgG}$ antibodies might presented in criticaly ill patients or various infections, including critical COVID-19 patients. Antiphospholipid antibodies activate endothelial cell and platelets, disrupt natural anticoagulant and fibrinolytic systems by interfere the functions of activated protein $\mathrm{C}$, protein $\mathrm{S}$, and tissue plasminogen activator, resulting in a procoagulant state which cause thrombotic events [6-8].

Previous studies showed markedly raised D-dimers, which defined as three-four fold increase, DIC, and ARDS were associated with increased mortality in COVID-19 infection $[9,10]$. These conditions were presented with our patient, which were hypothesized as the underlying causes of the ecchymosis. Therefore, the presence of ecchymosis in COVID-19 patients is indicating an occuring coagulopathy and associated with increased mortality in COVID-19 infection which is consistent with previous studies [9-11]. Due to increased mortality in the occurence of coagulopathy in COVID-19 infection, ISTH recommended prophylactic dose low molecular weight heparin (LMWH) should be considered in all patients (including non-critically ill) who require hospital admission for COVID-19 infection, in the absence of any contraindications (active bleeding and platelet count less than $25 \times 10^{9} / \mathrm{L}$ ), as was done in this case $[9,10]$. To date, there are many therapeutic candidates suggested to control the vasculopathy in COVID-19 infection including anti-inflammatory and anti-thrombotic treatment, such as tocilizumab (IL-6 inhibitor), bosentan (endothelin-1 receptor blocker), systemic steroid, iloprost (prostacyclin receptor agonist), alprostadil (Prostaglandin E1), pentoxifylline, and bevacizumab (Vascular Endothelial Growth Factor inhibitor). However, further study and RCTs are needed for these therapeutic candidates [12-15]. This patient was indeed in critical condition because of the severe COVID-19, however fortunately, the patient gradually became stable and clinically improved until the discharge from hospital and during the follow up.

Author contributions Dr. Widysanto as supervisor, consultant, and data collector; Dr. Wahyuni as supervisor, consultant, and data collector; Dr. Simanjuntak as supervisor, consultant, and data collector; Dr. Sunarso as supervisor, consultant, and data collector; Dr. Siahaan as supervisor, consultant, and data collector; Dr. Haryanto as supervisor, consultant, and data collector; Dr. Pandrya as supervisor, consultant, and data collector; Dr. Aritonang as supervisor, consultant, and data collector; Dr. Gunawan as main writer and data collector; Dr. Angela as main writer and data collector.

Funding None.

Data availability Data transparancy available.

\section{Compliance with ethical standards}

Conflict of interest All authors declare that they have no conflict of interest.

\section{References}

1 Manalo IF, Smith MK, Cheeley J, Jacobs R (2020) A dermatologic manifestation of COVID-19: transient livedo reticularis. J Am Acad Dermatol [Internet]. https://doi.org/10.1016/j. jaad.2020.04.018

2 Liu PP, Blet A, Smyth D, Li H (2020) The science underlying COVID-19: implications for the cardiovascular system. Circulation. https://doi.org/10.1161/CIRCULATIONAHA.120.047549

3 Magro C, Mulvey JJ, Berlin D, Nuovo G, Salvatore S, Harp J et al (2020) Complement associated microvascular injury and thrombosis in the pathogenesis of severe COVID-19 infection: a report of five cases. Transl Res. https://doi.org/10.1016/j. $\operatorname{trs} 1.2020 .04 .007$

4. Gupta N, Zhao YY, Evans CE (2019) The stimulation of thrombosis by hypoxia. Thromb Res 181:77-83 
5 Tolouian R, Zununi Vahed S, Ghiyasvand S, Tolouian A, Ardalan M (2020) COVID-19 interactions with angiotensin-converting enzyme 2 (ACE2) and the kinin system; looking at a potential treatment. J Ren Inj Prev [Internet] 9(2):19

6 Zhang Y, Xiao M, Zhang S, Xia P, Cao W, Jiang W et al (2020) Coagulopathy and antiphospholipid antibodies in patients with covid-19. N Engl J Med 382:e38. https://doi.org/10.1056/ NEJMc2007575

7 Makino J, Koshy S, Bajaj S, Jeong YG, Perlman DC (2017) Fulminant ecchymosis as the initial manifestation of antiphospholipid syndrome (APS) triggered by respiratory syncytial virus (RSV) infection: a case report and review of the literature. IDCases 7:19-22

8. Willis R, Pierangeli SS (2011) Pathophysiology of the antiphospholipid antibody syndrome. Autoimmun Highlights 2(2):35-52

9. Thachil J, Tang N, Gando S, Falanga A, Cattaneo M, Levi M et al (2020) ISTH interim guidance on recognition and management of coagulopathy in COVID-19. J Thromb Haemost 18(5):1023-1026

10. Tang N, Li D, Wang X, Sun Z (2020) Abnormal coagulation parameters are associated with poor prognosis in patients with novel coronavirus pneumonia. J Thromb Haemost 18(4):844-847
11. Casas D, Catal A, Fern D (2020) Classification of the cutaneous manifestations of COVID-19: a rapid prospective nationwide consensus study in Spain with 375 cases. Br J Dermatol 183(1):71-77

12 Almashat SA (2020) Vasculitis in COVID-19: a literature review. J Vasc 6(1):1-5

13 Moezinia CJ, Ji-Xu A, Azari A, Horlick S, Denton C, Stratton R (2020) Iloprost for COVID-19-related vasculopathy. Lancet Rheumatol 2(10):e582-e583

14 de Perosanz-Lobo D, Fernandez-Nieto D, Burgos-Blasco P, SeldaEnriquez G, Carretero I, Moreno C et al (2020) Urticarial vasculitis in COVID-19 infection: a vasculopathy-related symptom? J Eur Acad Dermatology Venereol 34(10):e566-e568

15 Becker RC (2020) COVID-19-associated vasculitis and vasculopathy. J Thromb Thrombolysis 50(3):499-511

Publisher's Note Springer Nature remains neutral with regard to jurisdictional claims in published maps and institutional affiliations. 